\section{Paradigms of Oxygen Therapy in Critically III Patients}

\section{Abstract}

Oxygen administration to patients has potential advantages and some evident drawbacks.

Several clinical settings, mainly critical scenarios, demand high oxygen inspired fractions $\left(\mathrm{FiO}_{2}\right)$ in order to assure safe reserve margins for an eventual arterial desaturation (for example before tracheal intubation in operating room).

On the other end some evidence shows that maintain high $\mathrm{FiO}_{2}$ during surgical procedures and/or controlled ventilation in the intensive care units might be more harmful than beneficial.

The aim of this revision was to show what type of decision clinicians may make, regarding the amount of oxygen that should be given to patients, with a special focus on the pros and cons of its liberal utilization.

Keywords: Oxygen toxicity; Oxygen therapy; Atelectasis

Received: December 09, 2016; Accepted: December 20, 2016; Published: December 27, 2016

\section{Introduction}

Oxygen has been present in the atmosphere for 5 billion years. At the beginning, it was present in a low concentration, but nowadays is the second most common gas $(\approx 21 \%)$, preceded only by nitrogen ( $\approx 78 \%)$. It is well known that oxygen is necessary for life maintenance. Oxygen acts as final electron acceptor in the production of adenosine triphosphate (ATP), which is the main source of energy in our body [1].

It was in 1772 that Scheele discovered oxygen although Priestley has published his findings first, in 1775. Priestley was the first to suggest that oxygen could have adverse effects. Later in 1878, Bert described oxygen neurotoxicity when noticed that high-pressure oxygen caused convulsions in larks. Smith observed that rats developed pneumonia after being exposed to high concentration of oxygen for 4 days [2]. Oxygen toxicity has been studied for many years and two mechanisms by which oxygen may cause its adverse effects have been proposed: The production of free radicals, which lead to the disequilibrium between oxidative and anti-oxidative species; and cell metabolism modification and enzymatic inhibition [3].

We know that oxygen is essential for survival but it can be toxic if it is used in excess. This raises the question: How much oxygen
Marta Carvallho ${ }^{1}$, Maria Soares ${ }^{1}$ and Humberto S Machado $0^{1,2}$

\author{
1 Serviço de Anestesiologia, Centro \\ Hospitalar Universitário do Porto, \\ Portugal \\ 2 Instituto de Ciências biomédicas Abel \\ Salazar, Universidade do Porto, Portugal
}

\section{Corresponding author: Humberto S Machado}

\section{Đhjs.machado@gmail.com}

MD, M.Sc.. PhD, Serviço de Anestesiologia, Centro Hospitalar Universitário do Porto, Largo Professor Abel Salazar, 4099-001 Porto, Portugal.

Tel: 351935848475

Citation: Carvalho M, Soares M, Machado HS. Paradigms of Oxygen Therapy in Critically III Patients. J Intensive \& Crit Care 2017, 3:1.

can we use without inducing lesion? Many factors should be considered in order to respond to this question. In this review, we describe and discuss the pros and cons of using supplementary oxygen.

\section{Pros}

The use of high fraction of oxygen inspired $\left(\mathrm{FiO}_{2}\right)$ augments the tolerance time to apnea. In anesthesia practice this is important especially during the induction and emergence of anesthesia. The aim of pre-oxygenation is to replace all the nitrogen in the function residual capacity (FRC) for oxygen. This increases the oxygen stores available and, therefore, increases tolerance to apnea time [4]. In addition, it is important to maintain airway patency and exposure to high $\mathrm{FiO}_{2}$ to permit the oxygen diffusion to the alveoli during the anesthesia induction. This phenomenon is called apneic oxygenation [5,6]. Edmark et al. [7] evaluated atelectasis formation and time to desaturation in patients exposed do $\mathrm{FiO}_{2}$ of $60 \%, 80 \%$ and $100 \%$ during anesthesia induction. They concluded that induction with $80 \% \mathrm{FiO}_{2}$ causes 
minimal atelectasis but the time to desaturation was significantly shortened compared to $100 \% \mathrm{FiO}_{2}$.

It has been proposed that high $\mathrm{FiO}_{2}$ reduces incidence of surgical wound infection [8-10]. Surgical-wound infections (SWI) are common and can be a serious problem with implications in morbidity and mortality. The oxidative process is essential in the defense against pathogens. Greif et al. [8] included 500 patients proposed to colorectal surgery and showed that exposure to 80 $\mathrm{FiO}_{2} \%$ during anesthesia and $2 \mathrm{~h}$ post op reduce the incidence of SWI when compared to exposure of $30 \% \mathrm{FiO}_{2}$.

In 2009, a meta-analysis supported these results by showing that supplemental perioperative oxygenation is beneficial in preventing SWI in patients undergoing colorectal surgery [9]. More studies may be needed to determine if the use of supplemental oxygen is beneficial for patients undergoing other types of surgery. The use of $100 \% \mathrm{FiO}_{2}$ during perioperative period seems to increase antimicrobial and pro-inflammatory responses of the alveolar macrophages during anesthesia.

Kotai et al. [11] suggested that these alterations may be beneficial for immune defense in lung tissue.

The effect of high concentration of $\mathrm{FiO}_{2}$ on postoperative nausea and vomiting (PONV) is still not clarified as previous studies had divergent results. In 2013, a meta-analysis including 11 studies regarding the impact of high $\mathrm{FiO}_{2}$ and PONV showed a weak beneficial effect on nausea [10].

Despite the increase of atelectasis formation with exposure to high $\mathrm{FiO}_{2}$, there are studies showing that this have no impact in pulmonary function. Besides that, it has been suggested that there is no difference in the incidence of pulmonary complications between those who were exposed to high $\mathrm{FiO}_{2}$ and low $\mathrm{FiO}_{2}$ during perioperative period [12].

Finally, the work of Lambertsen and his colleagues at the University of Pennsylvania has shown the relationship between inspired oxygen partial pressures and the time for the appearance of pulmonary and central nervous system toxicity symptoms. It seems that neurologic toxicity occurs only when oxygen exposure happens at high pressure and pulmonary toxicity appears when exposure to high $\mathrm{FiO}_{2}$ oxygen is prolonged in time [13].

\section{Cons}

It is very important to carefully decide on the level of inspired fraction that should be used during Oxygen is needed in order to assure patient safety in critical moments, but excessive amounts of oxygen inspired fractions might induce physiologic lesions. It is well recognized and confirmed by recent publications that hyperoxia may induce pulmonary toxicity and cell damage mediated by radical oxygen species (ROS). High doses of oxygen have also been associated to target-organ damage, mainly brain and heart, decreased cerebral and coronary blood flow, systemic vasoconstriction and decreased cardiac output.

High oxygen inspired fractions have been correlated with pulmonary toxicity induced by several mechanisms, including increased inter-cellular adhesion molecules, neutrophil recruitment, 8-iso PGF2 alpha, nitrous oxide, and synthesis of collagen-I and monocyte chemotactic protein $1[14,15]$.

Davis et al. [16] evaluated early reversible changes in human alveolar structures induced by hyperoxia. The authors evaluated 14 normal subjects by broncho alveolar lavage before and immediately after they had breathed more than 95\% oxygen for $16.7 \pm 1.1 \mathrm{~h}$. They concluded that although some of the effects of oxygen exposure for $17 \mathrm{~h}$ were reversible, hyperoxia for this short time period can decrease the structural or functional barriers that normally prevent alveolar-capillary leak and induce processes that may culminate in fibrosis of the alveolar wall.

Regarding toxicity induced by oxygen, Machado et al. [17] conducted an experimental prospective randomized study to evaluate lung inflammation with oxygen supplementation in tracheotomized spontaneously breathing rabbits. Rabbits were randomly assigned to 4 groups: groups 1 and 2 were submitted to $0.5 \mathrm{~L} / \mathrm{min}$ oxygen supplementation, for 20 or 75 min, respectively; groups 3 and 4 were left at room air for 20 or $75 \mathrm{~min}$. The results showed that oxygen supplementation in spontaneous breathing is associated with an increased inflammatory response when compared to breathing normal room air, and this inflammatory response was mainly constituted by polymorphonuclear cells [17].

Another physiologic change that can be induced by high oxygen inspired fraction is the development of absorption atelectasis. Atelectasis may develop with pre-oxygenation with $100 \% \mathrm{FiO}_{2}$ [18]. This is a major issue and needs a special intervention, namely the use of positive end expiratory pressure (PEEP) to maintain the lungs open [18] as even recent investigation has shown that if this is not instituted to the patient, the lung will certainly be poorly aerated [19].

Hedenstierna [20] has shown that patients whose anesthesia was induced during pre-oxygenation with $100 \% \mathrm{O}_{2}$ for $3 \mathrm{~min}$ had atelectasis, on average, in $10 \%$ of the lung area (corresponding to $15-20 \%$ of lung tissue). Pre-oxygenation with $80 \% \mathrm{O}_{2}$ for a similar time caused much less atelectasis, with a mean of $2 \%$. Reducing inspired oxygen to $60 \%$ during pre-oxygenation almost eliminated atelectasis and nothing was observed with $30 \% \mathrm{O}_{2}$. Also, the duration of pre-oxygenation with $100 \%$ oxygen can affect the amount of atelectasis that is produced. Thus, preoxygenation with $100 \% \mathrm{O}_{2}$ for 6 min resulted in a mean of $6 \%$ atelectasis, whereas $100 \% \mathrm{O}_{2}$ for shorter time of ventilation ( 3 $\mathrm{min}$ ) resulted in a little more than $2 \%$. Finally, 30\% oxygen for 3 min resulted in barely visible atelectasis of around $0.3-0.4 \%$. The focus anesthetic regime should be to deliver a patient with no atelectasis to the postoperative ward and to keep the lung open.

\section{In Stroke Patients}

Few studies have addressed supplemental oxygen therapy in stroke patients and the results are controversial. Rønning et al. [21] performed a study involving 550 stroke patients who were randomly assigned between two groups: to a group that received supplemental oxygen treatment (100\% atmospheres, $3 \mathrm{~L} / \mathrm{min}$ ) 
for $24 \mathrm{~h}(\mathrm{n}=292)$ or to a control group, which did not receive additional oxygen. Results showed that there was no statistically significant difference in one year survival between groups, but patients with less severe strokes (Scandinavian Stroke Scale $>40$ ) had a higher and significant survival rate in the control group (OR $0.45 ; 95 \% \mathrm{Cl} 0.23$ to $0.90 ; p=0.023$ ).

More recently, Roffe et al. [22] conducted a randomized clinical trial in 289 patients who suffered a stroke and were given supplemental oxygen therapy for $72 \mathrm{~h}$ after admission on the local stroke unit. They found a slight short-term improvement in the oxygen group regarding NIHSS at end of one week, but their findings may have been biased by the imbalance of stroke severity between the two groups.

Finally, The Stroke Oxygen Pilot Study did not find long-term significant differences in outcome among stroke patients submitted to supplemental oxygen therapy compared to standard of care [23].

There is a general consensus in what concerns the need for larger studies in this field, but the best current evidence states that oxygen therapy should not routinely be used in stroke patients and it might be deleterious in patients with minor strokes.

\section{In Resuscitation Following Cardiac Arrest}

A primary contributor to death and disability in resuscitated patients is the anoxic brain injury that typically follows a severe ischemia/reperfusion insult. To date, therapeutic hypothermia is the only proven treatment for anoxic brain injury after cardiac arrest [24-26]. However, recent evidence suggests that, in addition to body temperature, the amount of oxygen delivered to the brain after reperfusion may represent an important and modifiable factor in post resuscitation care. It is common to deliver supplemental oxygen in maximum concentrations during the resuscitative period, but this strategy may not be innocuous. Numerous laboratory investigations have identified a paradox relative to oxygen delivery to the injured brain. One large-scale clinical study has examined the relationship between excessive supplemental oxygen and outcome in post resuscitation patients [27]. Post resuscitation hyperoxemia exposure, defined as a partial pressure of arterial oxygen $\left(\mathrm{PaO}_{2}\right) 300 \mathrm{~mm} \mathrm{Hg}$, was found to be an independent predictor of in-hospital death. The mortality in patients with hyperoxemia was even higher than the mortality for patients with hypoxemia. Furthermore, hyperoxemia was associated with lower likelihood of independent functional status among patients who survived to hospital discharge [27].

Kilgannon et al. [28] designed a multicenter cohort study using the Project IMPACT database (intensive care units at 120 US hospitals) to better define the relationship between supranormal oxygen tension and outcome in post resuscitation patients. The proportion of in- hospital deaths was $41 \%$ for patients with a $\mathrm{PaO}_{2}$ in the range of 60 to $99 \mathrm{~mm} \mathrm{Hg}$ and rose to $65 \%$ for patients with a $\mathrm{PaO}_{2}$ in the range $400 \mathrm{~mm} \mathrm{Hg}$. The authors observed no clear single threshold of increased risk of death over ascending $\mathrm{PaO}_{2}$ ranges. In addition, they found a significant linear trend for the increase in mortality rates over ascending $\mathrm{PaO}_{2}$ ranges $(p<0.0001)$. The results of this multicenter study showed a linear dose-dependent relationship between supranormal oxygen tension and relative risk of in-hospital mortality, but no evidence supporting a single threshold for harm from supranormal oxygen tension [28].

\section{Acute Myocardial Infarction}

Oxygen is widely used in people with acute myocardial infarction (AMI) although evidence of its benefit is controversial. Many systematic reviews have concluded that there is insufficient evidence to know whether oxygen reduces increases or does not have an effect on heart ischemia or infarct size.

The rationale for supplemental oxygen in AMI is that it may improve the oxygenation of the ischemic myocardial tissue and reduce ischemic infarct size, morbidity and mortality. However, potentially harmful mechanisms include the paradoxical effect of oxygen in reducing coronary artery blood flow and increasing coronary vascular resistance $[29,30]$ reducing stroke volume and cardiac output and the consequent reperfusion injury from increased oxygen free radicals [31,32].

In 2013, a Cochrane meta-analysis reviewed 4 articles where oxygen supplemental therapy was used in over 400 non-hypoxic patients. When the data were pooled, twice as many people on oxygen died when compared to the group that was given air. Meta-analysis for mortality in participants with confirmed AMI: risk ratio (RR) 2.11 (95\% confidence interval $(\mathrm{Cl}) 0.78$ to 5.68); meta-analysis for mortality in an intention-to-treat population (including those who did not have AMI): RR 2.05 (0.75 to 5.58) [33].

However, due to the low number of deaths in all four studies (17 in total), the authors recognized that evidence is lacking power and results may have been influenced by chance. As of such, current evidence does not support or clearly refutes the use of supplemental oxygen therapy in non-hypoxic AMI patients.

\section{Neonatal Resuscitation}

Up to $10 \%$ of newborns require some degree of resuscitation, ranging from simple stimulation to assisted ventilation [34]. The aim of resuscitation is to prevent not only neonatal death but also the adverse long-term neurodevelopmental squeals associated with birth asphyxia [35].

Recommendations point out $100 \%$ oxygen as the gas to use during neonatal resuscitation. Nonetheless, many experts challenge this practice on the basis that little evidence exists to support it [35]. Increasing work of breathing and the increased generation of oxygen free radicals are amongst the potential adverse effects of supplemental oxygen [36].

Cochrane meta-analyses of five studies comparing $100 \%$ oxygen versus room air in neonatal resuscitation showed a reduction in mortality in the room air group, with no increase in future complications (including long-term neurodevelopmental outcome) [35]. However, long-term follow-up (18-24 months) was only obtained in one study and the proportion of eligible patients 
was under $70 \%$. Also, up to $25 \%$ of patients assigned to the room air group received back-up $100 \%$ oxygen therapy. The authors concluded that there is no evidence to recommend using room air over $100 \%$ oxygen or vice versa and that future research is required in this field.

\section{In Critical Care}

Oxygen administration is the most widely prescribed therapy in critically ill patients and frequently represents a life-saving intervention. Clinical data regarding the relationship between arterial hyperoxia and outcome are contradictory. The question whether exposure to supranormal arterial $\mathrm{O}_{2}$ tensions $\left(\mathrm{PaO}_{2}\right)$ is safe in critically ill patients remains unanswered.

In 2014, Damiani et al. performed a systematic review and metaanalysis of studies describing the relationship between arterial hyperoxia and mortality in critically ill patients [37]. The majority of studies that have explored the relationship between arterial hyperoxia and mortality in critically ill patients are retrospective observational investigations, with only one prospective beforeafter study, which supported the use of a more conservative strategy. The authors concluded that hyperoxia exposure may be associated with mortality in patient subsets (post-cardiac arrest, stroke and traumatic brain injury). However, they referred that these results must be interpreted carefully given the heterogeneity in criteria used for defining hyperoxia exposure and a significant inconsistency between study findings.

Panwar et al. conducted a multicenter randomized controlled trial to determine whether a conservative oxygenation strategy is a feasible alternative to a liberal oxygenation strategy among ICU patients requiring invasive mechanical ventilation (IMV). At four multidisciplinary ICUs, 103 adult patients deemed likely to require IMV for $\geq 24 \mathrm{~h}$ were randomly allocated to either a conservative oxygenation strategy with target $\mathrm{SpO}_{2}$ of $88-92 \%$ or a liberal oxygenation strategy with target $\mathrm{SpO}_{2}$ of $\geq 96 \%$. The study findings support the feasibility of delivering conservative oxygen therapy in patients on invasive MV as alternative to the usual oxygenation strategy, while being effective in reducing exposure to hyperoxia [38,39].

There is still insufficient evidence regarding the safety of arterial hyperoxia in critically ill patients. Most of the existing studies are observational investigations with highly heterogeneous characteristics and inconsistent results. Randomized controlled trials are lacking.

Arterial hyperoxia may be associated with higher mortality in some critically ill patient subsets (post-cardiac arrest, stroke and traumatic brain injury).

While a liberal use of oxygen may provide a margin of safety against hypoxia, a more conservative approach might reduce potentially harmful exposure to excessive $\mathrm{FiO}_{2}$, hyperoxemia and tissue hyperoxia.

\section{Conclusion}

The selection of optimum arterial oxygenation goals is essential if cellular hypoxia and unnecessarily excessive oxygenation (and ventilation) are to be avoided. It is imperative to balance the risks associated with hypoxemia and hyperoxemia forms during daily assessment of patients.

The traditional clinical approach to oxygen therapy has been to prioritize the avoidance of hypoxemia while being relatively tolerant of hyperoxemia. The possibility that "too much" oxygen may be as harmful as "not enough" should lead to a pragmatic rethinking of the practice of oxygen administration.

Perhaps, the most prudent and advisable practice when oxygen needs to be used, will be to think of it as a lifesaving drug with its undeniable advantages and uses, and at the same time, a gas that has to be handle with special care, thought that hypoxia may be managed by several means (PEEP, recruitment maneuvers) before the decision of a high-inspired fractions of oxygen should be always in our minds.

Given the widespread use of $\mathrm{O}_{2}$ therapy in critical care, clinicians should be aware of the potentially deleterious effects of excessive $\mathrm{O}_{2}$ administration. 


\section{References}

1 Cooper G (2000) The cell, molecular approach, $2^{\text {nd }}$ edition. Sinauer Associates, Sunderland.

2 Chawla M, Lavania C (2001) Oxygen toxicity. Medical Journal of Armed Forces India 57: 131-133.

3 Patel D, Goel A, Agarwai S, Garg P, Lakhani (2003) Oxygen toxicity. J Ind Acad of Cli Med 4: 234-237.

4 Sirian R, Wills J (2009) Physiology of apnoea and the benefits of preoxygenation. Continuing education in anaesthesia, critical care and pain 9: 105-108.

5 Hedenstima G (2014) Effects of anaesthesia on ventilation/perfusion matching. Eur J Anaesth 31: 447-449.

6 Edmark L, Kostova-Aherdan K, Enlund M, Hedenstierna G (2003) Optimal oxygen concentration during induction of general anesthesia. Anesth 98: 1.

7 Weingart S, Levitan R (2001) Preoxygenation and prevention of desaturation during emergency airway management. Ann of Emer Med 20: 4-11.

8 Greif R, Akça O, Horn E, Kurz A, Sessler D (2000) Supplemental perioperative oxygen to reduce the incidence of surgical wound infection. N Eng J Med 342: 161-167.

9 Al-Niaimi A, Safdar N (2009) Supplemental perioperative oxygen for reducing surgical site infection: A meta-analysis. J Eval Clin Pract 15: 360-365.

10 Hovaguimian F, Lysakowski C (2013) Effect of intraoperative high inspired oxygen fraction on surgical site infection, postoperative nausea and vomiting, and pulmonary function. Systemic review and meta-analysis of randomized controlled trials. Anesth 119: 303-316.

11 Kotani N, Hashimoto H (2000) Supplemental intraoperative oxygen augments antimicrobial and proinflammatory responses of alveolar macrophages. Anesth 93: 15-25.

12 Meyhoff CS, Wetterslev J, Jorgensen LN, Henneberg SW, Høgdall C, et al. (2009) Effect of high perioperative oxygen fraction on surgical site infection and pulmonary complications after abdominal surgery. The PROXI rabdomized clinical trial. J Ame Med Ass 302: 1543-1550.

13 Lambertsen C (1987) Definition of Oxygen Tolerance in Man" (Predictive Studies V). Institute for Environmental Medicine University of Pennsylvania Medical Center, Philadelphia.

14 Huang D, Fang F, Xu F (2016) Hyperoxia induces inflammation and regulates cytokine production in alveolar epithelium through TLR2/4-NF-kB-dependent mechanism. Eur Rev Med Pharm Sci 20: 1399-1410.

15 Kotani N, Hashimoto H, Sessler D, Muraoka M, Hashiba E, et al. (2000) Supplemental intraoperative oxygen augments antimicrobial and proinflammatory response of alveolar macrophages. Anesth 93: 3-5.

16 Bruce DW, Rennard S, Bitterman P, Crystal R (1983) Early reversible changes in human alveolar structures induced by hyperoxia. $\mathrm{N}$ Eng $\mathrm{J}$ Med 309: 878-883.

17 Machado H, Nunes C, Sa P, Couceiro A, Silva A, et al. (2014) Increased lung inflammation with oxygen supplementation in tracheotomized spontaneously breathing rabbits: An experimental prospective randomized study. BMC Anesth 14: 86.

18 Hedenstierna G (2014) Effects of anaesthesia on ventilation/ perfusion matching. Eur J Anaesthesiol 31: 447-449.
19 Borges JB, Porra L, Pellegrini M, Tannoia A, Derosa S, et al. Zero expiratory pressure and low oxygen concentration promote heterogeneity of regional ventilation and lung densities. Acta Anaesthesiol Scand 60: 958-968.

20 Hedenstierna G (2012) Oxygen and anesthesia: what lung do we deliver to the post-operative ward? Acta Anaesthesiol Scand 56: 1-11.

21 Rønning OM, Guldvog B (1999) Should Stroke Victims Routinely Receive Supplemental Oxygen? Stroke 30: 2033-2037.

22 Roffe C, Ali K, Warusevitane A, Sills S, Pountain S, et al. (2011) The SOS pilot study: A RCT of routine oxygen supplementation early after acute stroke-effect on recovery of neurological function at one week. PLOS ONE 6: e19113.

23 Ali K, Warusevitane A, Lally F, Sim J, Sills S, et al. (2013) The stroke oxygen pilot study: A randomized controlled trial of the effects of routine oxygen supplementation early after acute stroke-Effect on key outcomes at six months. PLoS ONE 8: e59274.

24 Hypothermia after Cardiac Arrest (HACA) Investigators (2002) Mild therapeutic hypothermia to improve the neurologic outcome after cardiac arrest. N Engl J Med 346: 549-556.

25 Bernard SA, Gray TW, Buist MD, Jones BM, Silvester W, et al. (2002) Treatment of comatose survivors of out-of-hospital cardiac arrest with induced hypothermia. N Engl J Med 346: 557-563.

26 Neumar RW, Nolan JP, Adrie C, Aibiki M, Berg RA, et al. (2008) Postcardiac arrest syndrome: Epidemiology, pathophysiology, treatment and prognostication: A consensus statement from the International Liaison Committee on Resuscitation (American Heart Association, Australian and New Zealand Council on Resuscitation, European Resuscitation Council, Heart and Stroke Foundation of Canada, InterAmerican Heart Foundation, Resuscitation Council of Asia, and the Resuscitation Council of Southern Africa); the American Heart Association Emergency Cardiovascular Care Committee; the Council on Cardiovascular Surgery and Anesthesia; the Council on Cardiopulmonary, Perioperative and Critical Care; the Council on Clinical Cardiology; and the Stroke Council. Circulation 118: 24522483.

27 Kilgannon JH, Jones AE, Shapiro NI, Angelos MG, Milcarek B, et al. (2010) Emergency medicine shock research network (EMShockNet) investigators. Association between arterial hyperoxia following resuscitation from cardiac arrest and in-hospital mortality. JAMA 303: 2165-2171.

28 Kilgannon JH, Jones A, Parillo J, Dellinger P, Milcarek B, et al. (2011) Relationship between supranormal oxygen tension and outcome after resuscitation from cardiac arrest. Circulation 123: 2717-2722.

29 McNulty PH, King N, Scott S, Hartman G, McCann J, et al. (2005) Effects of supplemental oxygen administration on coronary blood flow in patients undergoing cardiac catheterization. Am J Phys, Heart \& Circ Phys 288: H1057-H1062.

30 McNulty PH, Robertson BJ, Tulli MA, Hess J, Harach LA, et al. (2007) Effect of hyperoxia and vitamin C on coronary blood flow in patients with ischaemic heart disease. J Appl Physiol 102: 2040-2045.

31 Milone SD, Newton GE, Parker JD (1999) Hemodynamic and biochemical effects of $100 \%$ oxygen breathing in humans. Canadian Journal of Physiol and Pharmacology 77: 124-130.

32 Rousseau A, Bak Z, Janerot-Sjoberg B, Sjoberg F (2005) Acute hyperoxemia-induced effects on regional blood flow, oxygen consumption and central circulation in man. Acta Physiologica Scandinavica 183: 231-240. 
33 Cabello JB, Burls A, Emparanza JI, Bayliss S, Quinn T (2013) Oxygen therapy for acute myocardial infarction. Cochrane Database Syst Rev 21: CD007160.

34 Saugstad OD (1998) Practical aspects of resuscitating new-born infants. Eur J Ped 157: S11-S15.

35 Tan A, Schulze AA, O'Donnell CPF, Davis PG (2005) Air versus oxygen for resuscitation of infants at birth. Cochrane Database Syst Rev 2: CD002273.

36 Mortola JP, Frapell PB, Dotta A (1992) Ventilatory and metabolic response to acute hyperoxia in newborn. Am Rev Resp Dis 146: 11-15.
37 Damiani E, Adrario E, Giradis M, Romano R, Pelaia P, et al. (2014) Arterial hyperoxia and mortality in critically ill patients: A systematic review and meta-analysis. Crit Care 18: 711.

38 Panwar R, Hardie M, Bellomo R, Barrot L, Eastwood G, et al. (2016) Conservative versus liberal oxygenation targets for mechanically ventilated patients - A pilot multicenter randomized controlled trial. Am J Respir Crit Care Med 193: 43-51.

39 Machado $\mathrm{HS}$ (2016) $\mathrm{O}_{2}$ and $\mathrm{N}_{2} \mathrm{O}$ : Between the rock and the hard place. J Anesth Clin Res 7: 5. 